\title{
Collaborative Service Blueprinting for Design Thinking: Evaluation of a Digital Prototype
}

\author{
Tobias Potthoff ${ }^{1}$, Dominik Siemon ${ }^{2}$, Konstantin Wilms ${ }^{1}$, Sascha Möser ${ }^{2}$, Marco Hellmann ${ }^{1}$, \\ Stefan Stieglitz ${ }^{1}$, Susanne Robra-Bissantz ${ }^{2}$ \\ ${ }^{1}$ University of Duisburg-Essen, Germany \\ \{tobias.potthoff|konstantin.wilms|marco.hellmann \\ stefan.stieglitz\}@,uni-due.de \\ ${ }^{2}$ University of Braunschweig, Germany \\ \{d.siemon|sascha.moeser|s.robra-bissantz\} \\ @,tu-braunschweig.de
}

\begin{abstract}
A high degree of innovation is becoming a decisive factor for companies aiming at sustainable competitive advantages. New methods of innovation have become increasingly important in practice. Using such methods in globally operating teams requires location- and time-independent solutions such as web-based collaboration tools. This paper evaluates the ability to digitalize the service innovation method Service Blueprinting using a selfdeveloped prototype. The prototype is evaluated thoroughly in two different studies. In-depth user tests with Design Thinking experts and laymen prove the suitability for Design Thinking and the interaction with the prototype from a HCI perspective. Additionally, we evaluate the acceptance, perceived usefulness and effectiveness quantitatively in the light of the prototype's collaboration capability. The results indicate the transferability of the required mindset through the digital tool as well as its the acceptance and usefulness. It represents a practical and suitable solution for the digitalizing of Service Blueprinting.
\end{abstract}

\section{Introduction}

In an increasingly interconnected world, the global competition for customers and market share is growing. Companies are faced with immense challenges to resist international competition [41]. An elementary role in this context is played by innovations that create competitive advantages and secure sustainable success in globalized markets [23, 45]. In order to be able to better react to dynamic markets, companies need innovative products and services, as well as superior methods for their development [39]. In addition, it is essential to focus on the specific needs of the customer. This is one of the most important approaches to develop products and services that are successful on the market in a time of oversupply [22, 41]. Customer proximity, successfully practiced from the beginning of product development, thereby becomes a competitive advantage $[1,23]$.

As a result of these developments, more and more companies rely on new approaches to innovate, which include the customer in this whole process [47]. One of these approaches is Design Thinking (DT), an interdisciplinary, collaborative method for solving complex problems in the area of product and service innovation [32].

One particularly suitable method for the development of new services in the field of DT is Service Blueprinting [40]. It visualizes the process of a service and describes it within a defined basic structure from the customer's point of view [30]. Thus, weaknesses and optimization potentials in the process can be quickly identified and improved [3, 38]. Service Blueprinting is therefore ideal for the innovation of all kind of services [3]. As project teams are increasingly composed of employees who do not work at one place, but are globally dispersed [15], digital solutions that allow real-time collaboration over the internet are needed [14]. Individual team members can work together from almost any place in the world. This saves time and money, and also facilitates the digital processing, for example within a presentation [17].

This paper examines the potential of digitalizing DT methods using the example of Service Blueprinting. For this purpose, we followed the Design Science Research (DSR) approach and developed as well as evaluated a web-based prototype for collaborative Service Blueprinting. In the first place, we conducted in-depth user tests with DT experts and laymen to assess the appropriateness and usability. In a second evaluation step, we analyzed the broader user acceptance and motivational aspects quantitatively [27]. 
For a better understanding, the theoretical foundations of Service Blueprinting in the context of DT are presented next. On this basis, the web-based prototype was designed and implemented. Subsequently, we present the two evaluation studies. Finally, the results are discussed and implications for further research and practical use are given.

\section{Context and motivation}

This study is part of a research project in which we evaluate the digitalization potentials of multiple DT methods. The goal is to ultimately develop a webbased system that can be used to design innovative services. DT is primarily used to develop new products, services, processes or entire business models. However, it does not only contain the creative aspects of design, but also conceptual and technical design [32]. In this way, DT can be characterized as a method of innovation in the practice-oriented sense, with which complex problems of various kinds can be solved. According to Beyhl and Giese [2], DT contains of three levels: (1) DT describes a methodology in terms of a sequence of phases. (2) The phases have no fixed order and the specific sequence varies depending on the project. (3) Each phase contains methods which in turn can be assigned to individual or even several phases [32]. In this context, DT is understood as a universal and cross-departmental approach and is used in various fields of science and economy [32]. It is largely based on teamwork and collaboration and is, therefore, often applied in face-to-face meetings. As getting all participants to the same location in distributed teams can be expensive, web-based solutions are needed to save costs and time [17, 41]. These solutions often try to replace the analogue user experience with a digital representation [44, 46].

Service Blueprinting is a comprehensive method that can be applied in more than one stage of DT [40] and is therefore in the scope of our digitalization process. Service Blueprinting has already been implemented in a variety of software solutions that allow the digital creation of service blueprints. However, these solutions often do not allow collaborative work (e.g. real-time synchronization and team communication features), are not specifically for the creation of service blueprints (e.g. templates for digital whiteboards) or are not implemented in the context of DT. As collaboration is one of the key aspects of DT [32], we see high practical value for a comprehensive digital collaborative Service Blueprinting system in order to develop innovative services in dispersed teams [27]. While current literature has yet only focused on analog DT in group environments, digital group scenarios have mostly been ignored. Yet, there is a lack of research in terms of the utilization of digital methods within the DT sequence. In addition, we identified a research gap in scientific examinations of digital Service Blueprinting software, especially towards its usefulness, effectiveness and acceptance. Although Service Blueprinting has proven to be an effective method to innovate services $[3,33]$, the effectiveness of digital collaborative implementations have not been examined yet. In order to enable the detailed analysis of usage and collaboration behavior in a Service Blueprinting solution, only the development of an own prototype was expected to provide the best data basis for required analyses.

\section{Service Blueprinting}

Service Blueprinting is a method by which a service process can be visualized and described [3, 38]. Each aspect of the service is included from the point of view of the customer, the service provider and other involved parties $[3,30]$. The focus is on the customer and his/her interaction with the service provider. A service blueprint provides an overview of the entire service, so that weaknesses and problems in the process can be identified quickly. In addition to the individual process steps, a service blueprint also includes the interfaces between customer and service provider [3, 30, 33]. Service blueprints are simple in their representation, so that the method can be quickly learned and used [3]. Due to the clear structure, ideas can be rapidly visualized and developed collaboratively. This makes the method very suitable to use in a DT context. It usually consists of five levels, in which the individual steps of the service process are visualized.

In addition to the development of a service, Service Blueprinting also serves as an illustration for the internal and external employees of the service provider $[3,30]$. They can always look at the entire process of the customer and can therefore interact more precisely with him/her due to the clear presentation. Furthermore, a service blueprint serves the identification of future market potentials and thus promotes the innovation of the service [3]. Service Blueprinting has already been used in numerous service areas. For example, it was used to redesign a university enrolment and registration process [3], improve police work [40] or to optimize a university library [33]. 


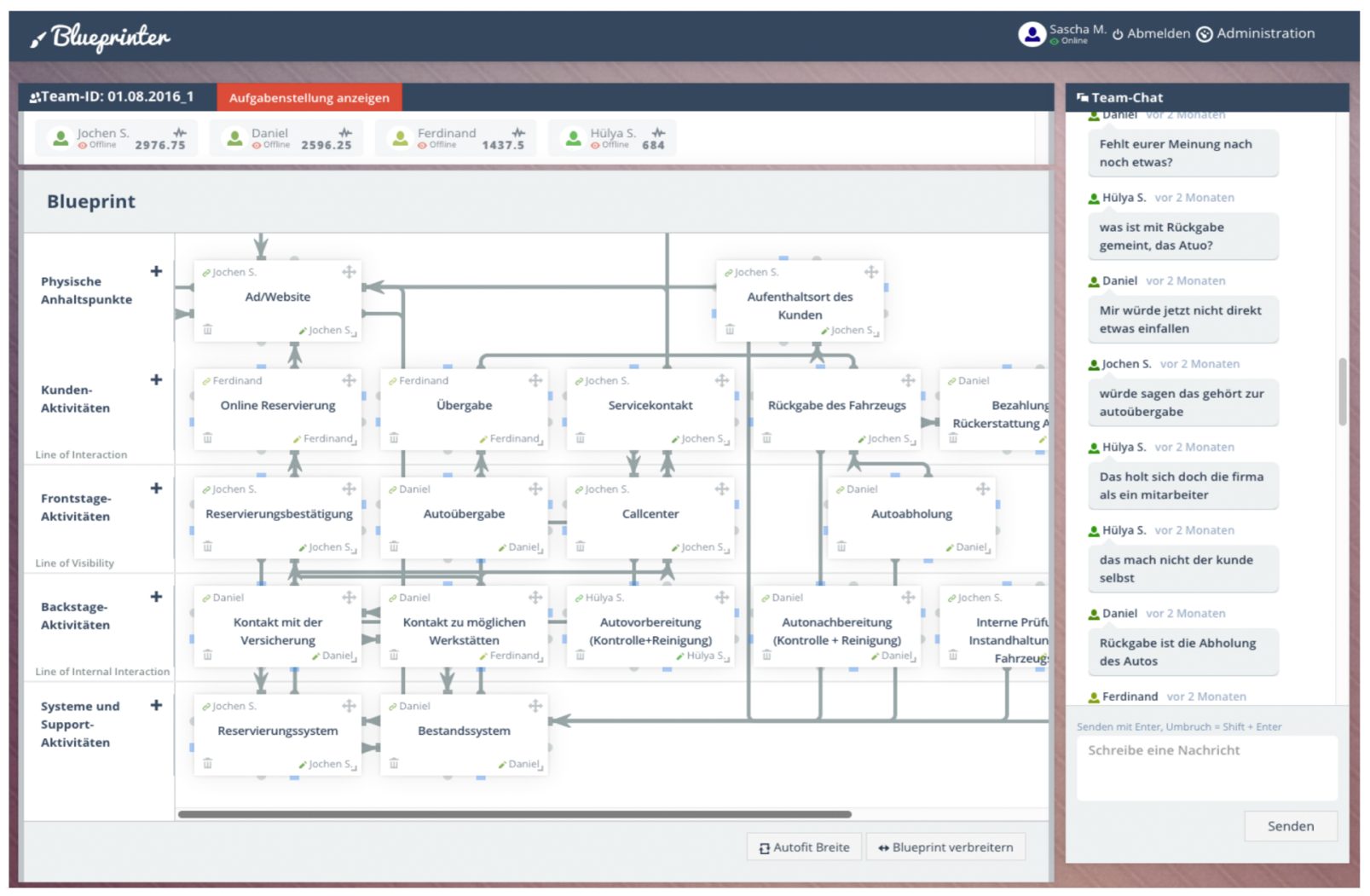

Figure 1. Screenshot of the prototype's main user interface (prototype in German)

\section{Web-based prototype for collaborative Service Blueprinting}

We followed the Design Science Research (DSR) approach to create new insights and results through the design of an innovative artefact [18]. Principles, practices and procedures of the methodology incorporate a consistent research, involving the construction of an innovative artefact and the evaluation of its effectiveness to contribute to a significant knowledge [18]. We identified a substantial research gap and diverse practical problems (e.g. the absence of software implementations of collaborative Service Blueprinting) and defined objectives for a solution. In this next section, we will present a solution in the form of a web-based prototype (artefact), which we designed and implemented. Subsequently, we evaluated the artefact in the context of DT to demonstrate its effectiveness towards our identified problems. We position our research as Improvement according to the DSR Knowledge Contribution Framework [16].

In the first step on the way to a full-fledged web application for Service Blueprinting, we built a

\footnotetext{
${ }^{1}$ React (or ReactJS) is an open-source JavaScript library to render data as HTML (https://facebook.github.io/react/).
}

prototype. With this web-based prototype, multiple users can simultaneously and collaboratively develop a service blueprint, where all actions are synchronized in real-time. Due to the high demands on the prototypes interactivity and reactivity, it is implemented as a single page application. This means that the entire page does not have to be reloaded every time a user interacts with it and therefore responds faster. The user interface of the prototype is based on the React library ${ }^{1}$. It is used to develop complex user interfaces with quickly changing data underneath. This makes React an ideal candidate for a fast-paced and collaborative web application [12]. A design pattern often used in conjunction with React, is Flux. The more specified implementation Redux ${ }^{2}$ is widely used in the React environment and is also used in our prototype [24].

The core element of the prototype is the blueprint editor for the collaborative creation of service blueprints. Figure 1 shows the basic structure of the user interface and depicts a completed blueprint. The main part of the interface is the blueprint editor with a main area, scrollable in horizontal direction. In this way, even very complex and therefore wide service blueprints can be displayed in a small space. Above

\footnotetext{
${ }^{2}$ Redux is a predictable state container for JavaScript apps (http://redux.js.org/).
} 
the editor is a list of all team members. A chat is placed on the right, where users can communicate with each other. Within the individual blueprint layers, users can create as many content elements as they need to create the service blueprint. All created blueprint elements can be freely moved and resized by dragging and dropping within the blueprint. This allows an intuitive customization of the layout and eases collaboration. All changes are automatically synchronized in the background, so that collaboration is achieved in realtime. While working on the service blueprint the chat interface is permanently available for communication within the team.

Such a digitization makes sense, only if the prototype proves to be appropriate and is accepted by users. The effectiveness of the collaboration also plays a decisive role. Following DSR, we include two thorough evaluation steps in the research endeavor.

\section{Study I: Qualitative user test}

Hewett et al. [19] basically differ between three areas of investigation in human-computer-interaction research: (1) We can look at the artefact's (e.g. software) application context and the to be performed tasks. Only if the artefact fulfills requirements from practice, an enduring and effective application will take place. (2) Another area are human factors like (cognitive) information processing, learning processes and the subjective satisfaction of users while using the software. Only if the user is subjectively satisfied, s/he will be willing to continue using the tool. (3) Hewett et al. further see a focus at the technical functionality and control elements (e.g. user interface). The effect of technical elements on the user is of special interest for our study because they are deemed as essential for the perceived usability [19].

Like mentioned, users also need to have a specified mindset in terms of a mental attitude and orientation to work effectively in a DT context [48]. This includes empathy, integrative thinking, fondness of experimenting, team orientation and optimism or motivation at work. Traditionally, DT supports the mindset by means of the open working environment $[32,35]$. In the context of digital DT, the software becomes responsible for the formation of relevant mental orientation. These considerations led to the first research question RQ1: Does the prototype support the required mindset of Design Thinking?

The method Service Blueprinting is suitable to structure service processes and to create a common understanding for each involved person. Furthermore, a service blueprint is considered as a living document, which is revised regularly [40]. Therefore, we assume that the application of the prototype can be used during
DT phases to structure information (e.g. Understand) and creative phases (e.g. Prototyping). However, this should be answered with $\boldsymbol{R Q 2}$ : Can the prototype be used in different Design Thinking phases?

Whether a tool is used in general or for a specific task depends on both, the user's judgement and the human computer interface. The subjective satisfaction of results, experienced user performance [20] and perceived convenience during use [26] is crucial. Convenience is defined by usability criteria, correctness and absence of negative feelings. Hence, we pose RQ3: Can the prototype evoke subjective satisfaction?

Like usual in DT, we expect the prototype to be used by people with different IT knowledge and DT experience. Therefore, it is of interest to examine how the design of the human computer interface affects the ability to learn both the software usage and the underlying DT method Service Blueprinting. The last research question in this user test is $\mathbf{R Q 4}$ : Can the prototype be easily learnt and does it enable the learning of the method Service Blueprinting?

\subsection{Study structure}

We used individual user tests to investigate the research questions. The involved participants did not know the prototype before which is required for outof-the-box-test and works well for the analysis of a software's ease of use. The participants had to deal with three different kinds of tasks. After each task, they were asked for relevant aspects defined in a guideline. With each task, the complexity was increased and the participants had to use more functions of the prototype. The tasks were: 1) The participants needed to digitize a printed/analogue Blueprint; 2) They needed to systematically create a service blueprint out of a textual description; 3) An existing service blueprint needed to be revised creatively. After each task, the participants were asked about their experiences in a semi-structured interview. Semi-structured interviews were most suitable for the analysis, since this type of interview allows a change of order and additional questions if it seems necessary.

The six participants were separated by their experiences with DT. We differentiated between four DT laymen (A-D) and two DT experts (E and F). The former did not know anything about the method service blueprinting nor anything similar outside of DT. Still, it was important that all participants have basic knowledge with web applications. All participants needed to basically understand service processes which was ensured in the recruitment. We coded and analyzed the observations and interview according to Mayring's qualitative content analysis 
[25]. Due to this method, the material is generalized in the first place and then reduced during an abstraction process. The central aspect of qualitative content analysis is the development of a categorical system which helps to identify the aspects that seem necessary for answering the research question from the wealth of the interview material. The validity of the first coding process was tested with two more coders.

\subsection{Results}

First, we found indications for the necessary DT mindset (RQ1). The participants stated to take the perspective of a customer while solving the task (e.g. interview A: "Basically, I went through a hotel reservation process from a customer's perspective"). We also observed integrative and systematical thinking. Like suggested for Service Blueprinting, the level of customer activities became the anchor in the digital prototype as well. The other layers were orientated towards this customer layer. Still at some points, the users expressed problems with the clarity of the created service blueprint which led to less information in the blueprint than provided and intended by the users. In particular, the last (creative) task 3 suffered from the lack of clarity because the users stopped improving the innovative ideas prematurely (e.g. interview E: "Directly with the first object, it [the blueprint] became unclear so that I got frustrated"). Also, the motivation of the participants was negatively influenced by usability problems. In this first study, we did not investigate the collaborative functions of the prototype yet. These will influence the DT mindset as well. We further asked whether the participants missed the possibility to vary symbols (e.g. color, shape etc.) since different colors and symbols are important in traditional DT. Still, the interviewed users did not see a need for more variety - at least to solve the given tasks.

Regarding RQ2 (support of different DT phases), the literature and theory basis suggest Service Blueprinting as a method for potentially all DT phases [40]. The participants were able to develop an as-is blueprint quickly which can be used in the beginning DT phases as a common basis for further discussions. Also, service blueprints can serve as to-be prototypes and as basis to check (with the customer) whether the newly designed process is sufficient and viable. Still, a creative revision of an existing process (task 3 ) was unsatisfactory because of the quickly arising lack of clarity and a difficult handling of more complex blueprints. The experts were not sure whether the tool is suitable for the ideating phase (interview F: "most tools are not as enjoyable [as an analogue method]").
This could hinder creativity. Interviewee $\mathrm{C}$ added: "With a piece of paper you are somehow freer."

We assume that the judgement about meaningful application areas of the tool is related to the subjective satisfaction. Especially the creative editing of an existing blueprint was seen as difficult due to usability issues. This insight coincides with RQ3 about subjective satisfaction. The participants stated to be satisfied with the results for the task 1 and 2. Still, they remained unsatisfied for task 3 with room for improvement. The participants considered the solving of task 1 and 2 as comfortable while task 3 caused discontent.

The satisfaction with the first and second tasks' results supports a high learnability of the software handling which we specifically addressed with RQ4. The participants stated to have not needed the integrated help feature. Instead, most symbols (e.g. for adding or moving elements) were self-explaining. In rare cases, the participants had problems to establish connections in the blueprint due to wrong interpretations of symbols. Minor problems like these occurred rarely and did not repeat once the participants identified their mistakes (e.g. interview B: "I think it's a matter of practice"). Though all participants learned how to use the software, they still had problems to understand the method of Service Blueprinting. It was especially difficult for those to define and differentiate the layers available in the service blueprint template. Concluding RQ4, we found support that participants had mostly no problem with the software or learnt how to use it quickly. Still, the software needs to better teach the method Service Blueprinting.

\section{Study II: Quantitative assessment}

In the second study, we focused on the motivational state in a collaborative scenario in which users had to virtually interact through the software prototype. One of the most important influencing factors of human behavior in different contexts is motivation. In general, a distinction is made between intrinsic and extrinsic motivation [43]. Intrinsic motivation refers to the inner impulse to perform an activity, for instance fun, a perception of sense or interest [36]. Extrinsic motivation, is a motivation triggered by external stimuli to pursue an activity. Both intrinsic and extrinsic motivation influence the extent to which digital technologies are used and accepted by users [43]. Intrinsic motivation of the users reflects the extent to which the application is used on its own, making it an important basis for the general willingness to use the prototype in a team environment. To be an alternative to the analogue use of Service Blueprinting, the prototype should therefore 
be able to motivate the users intrinsically. Hence, the first research hypothesis to be tested is H1: The prototype is capable of intrinsically motivating users to collaboratively create service blueprints.

A further criterion for the general suitability of the prototype is the users' technology acceptance. It describes the extent to which a user is generally willing to use a system. The perceived usefulness and the perceived ease of use play a decisive role in this respect [5]. The perceived usefulness expresses to what extent the user feels that the system increases her/his work performance. On the other hand, the perceived ease of use shows whether the user has the impression that the system can be used without physical and mental efforts [5]. In order to test this requirement, the following research hypothesis was formulated $\boldsymbol{H 2 :}$ : The prototype is perceived as useful and user-friendly.

Collaboration between multiple users only works when ideas can be effectively communicated and evaluated [10, 13]. This is an important requirement for the digital implementation of DT methods. For the prototype to be a useful tool for the collaborative creation of service blueprints in the long term, it must be perceived as effective by users. This applies both to the creation and evaluation of ideas as well as to the inclusion of all users. This requirement resulted in the third hypothesis H3: The users perceive the collaborative creation of service blueprints using the prototype as effective.

A main feature of our developed prototype is realtime collaboration, which ensures that every user can work at the same time on the service blueprint. Besides assessing the motivation and effectiveness of the team performance, we additionally aimed to measure the actual collaborative effort of each team member. Collaboration has a long history and can be explained as the joint effort towards common goals [29]. Collaboration occurs in any context where individuals seek to create value together [34]. The two main aspects of collaboration are (1) a common goal and (2) the joint effort of a collective. The common goal is an essential aspect of collaboration that motivates team members to work together. However, individuals in teams often tend to be social loafers or free-riders by not contributing to the team task [21]. Social loafing is the tendency of individuals expending less effort when working in a group than working individually, whereas free-riding means that individuals tend to contribute less if other team members are already contributing [11]. Therefore, we measure the activity of each team member by quantitatively accumulating every contribution to the team performance. This ensures that the prototype is not only perceived as an effective way to digitally create service blueprints, but additionally demonstrates an effective way to work in a collaborative manner.

These hypotheses are subsequently examined for their validity with respect to the developed prototype.

\subsection{Study structure}

The evaluation was carried out within the scope of a prototype test. A total of 44 subjects, aged between 23 and 65 , participated in the test $(M=29.09, S D=$ 10.40), of which 28 were male and 16 female participants. The participants were either students or working professionals. All participants were recruited during a lecture (participation was not compulsory). To examine the collaboration effectively, the test subjects were divided into a total of ten teams with four to five participants, who had to develop a service blueprint of a fictitious service. All teams were given an identical task, which they had to work on for 20 minutes. The task was to use the prototype to collaboratively develop a blueprint for a car rental company with a pick-up and drop-off service. All necessary information was given textually.

The test was carried out in groups in single sessions and all subjects participated from different locations. Prior to the start, all participants received a basic introduction to the Service Blueprinting method. In this way, no previous knowledge was required to participate. During the creation of the service blueprints, the subjects worked independently and communicated via the team chat. After 20 minutes, the prototype test ended and the subjects were automatically forwarded to the post-testing survey, which will be explained in the next section.

\subsection{Measurements}

The post-testing survey consisted of four demographic general items and 34 items on the evaluation of the prototype. The survey was structured as a self-report. In doing so, the participants responded individually on the basis of their own perception without the influence of the researcher [31]. In the design of the survey, we focused on widely-used and established constructs. The intrinsic motivation of the participants as a decisive factor for the use of our webbased artefact was investigated using the construct of the Intrinsic Motivation Inventory (IMI) [7]. In the original, the construct consists of seven individual measurements with a total of 45 items. We selected the four measurements Interest/Enjoyment, Effort/ Importance and Value/Convenience, which consist of seven items each $[7,36]$.

In addition, we examined the extent to which the prototype is accepted as a new technology. For this 
purpose, the Technology Acceptance Model was used [6]. For our evaluation, we selected the two main measurements Perceived Usefulness and Perceived Ease of Use with six and five items that aim to measure the degree to which the subjects believe that using the system would enhance their work performance and that using the system is free of effort [6].

In contrast to that, the last analyzed construct was the Perceived Effectiveness of the users. It indicates whether the subjects have recognized the collaboration, the interaction and the communication within the team as effective. At the same time, an assessment of the effectiveness of the collaboration process compared to a face-to-face meeting is assessed. The four items of this measurement have been taken from existing research on similar questions $[10,13]$. All items were assessed with a 7-point Likertscale that allows for more diverse responses and thus, permitting a more precise evaluation.

In addition to the survey, activity points of all subjects were calculated during the prototype testing. This includes the number of blueprint elements and connections created as well as the number of chat messages, as a measurement of user activity and collaboration. When working on the service blueprint, the points were weighted differently depending on the activity.

\subsection{Findings}

The data was analyzed using the software IBM SPSS Statistics. Subsequently, quantitative values such as the number of chat messages, blueprint elements, connections, log entries and activity points were transferred manually from the administration area of the prototype to SPSS. The internal consistency of the measurements is measured using the reliability coefficient Cronbach's alpha $\alpha_{c}$. Except of the measurement Effort/Importance $(\alpha=.576)$, all measurements score higher than $(\alpha \geq .826)$ and can thus be considered as consistent measurements of the concept. In Table 1, the results of the evaluation are presented with the mean values and standard deviations. Based on these, the hypotheses generally related to the suitability of the prototype can be evaluated. To calculate to what extent the mean values of the individual measurement deviate from the mean value of the 7-point Likert scale, a one sample t-Test was computed against the 7-point Likert scale mean value of 4 . The one sample t-Test is used to determine whether the mean value is statistically different from a known test value. The test can only be used to compare a sample mean to a specific constant, which in our case is the sample neutral point [4]. The data shows that the $p$-value is considerably lower then a significance level of five percent $(p<.05)$ for all measurements. Hence, all values deviate significantly from the mean value of the Likert-scale. Considering the mean values of each measurement, a significant positive deviation is shown for all measurements (see Table 1).

Table 1. Results of the survey from all subjects

\begin{tabular}{|l|c|c|c|c|c|c|}
\hline \multicolumn{9}{|c|}{} & \multicolumn{2}{|c|}{$t$-Test } \\
\hline Measurements & $M$ & $S D$ & $\alpha_{c}$ & Items & $t$ & $p$ \\
\hline $\begin{array}{l}\text { Interest / } \\
\text { Enjoyment }\end{array}$ & 4.955 & 1.049 & .882 & 7 & 6.035 & $\begin{array}{l}3.255 \\
* e^{-7}\end{array}$ \\
\hline $\begin{array}{l}\text { Effort / } \\
\text { Importance }\end{array}$ & 5.336 & .941 & .576 & 5 & 9.422 & $\begin{array}{l}2.290 \\
* e^{-4}\end{array}$ \\
\hline $\begin{array}{l}\text { Value / } \\
\text { Usefulness }\end{array}$ & 4.828 & 1.365 & .912 & 7 & 4.023 & $\begin{array}{l}5.096 \\
* e^{-12}\end{array}$ \\
\hline $\begin{array}{l}\text { Perceived } \\
\text { Usefulness }\end{array}$ & 4.894 & 1.331 & .918 & 6 & 4.454 & $\begin{array}{l}5.900 \\
* e^{-5}\end{array}$ \\
\hline $\begin{array}{l}\text { Perceived } \\
\text { Ease of Use }\end{array}$ & 5.550 & 1.095 & .875 & 5 & 9.392 & $\begin{array}{l}5.591 \\
* e^{-12}\end{array}$ \\
\hline $\begin{array}{l}\text { Perceived } \\
\text { Effectiveness }\end{array}$ & 4.460 & 1.210 & .826 & 4 & 2.522 & .0154 \\
\hline
\end{tabular}

Table 2 shows the activity points as well as the chat messages for every user as a measurement for the individual contributions. The log entries per person are composed of the aggregated number of created blueprints, established connections between the blueprint elements and the chat messages. It, therefore, also measures the individual performance. In addition, the number of blueprint elements and the number of element connections per team represent the team performance for the ten teams of our experiment.

Table 2. Quantitative results of all teams

\begin{tabular}{|l|c|c|}
\hline Measurements & $M$ & $S D$ \\
\hline Chat messages per person & 14.03 & 5.038 \\
\hline Blueprint elements & 22.30 & 2.751 \\
\hline Element connections & 17.50 & 7.075 \\
\hline Log entries per person & 41.89 & 7.124 \\
\hline Activity points per person & 1857.75 & 317.49 \\
\hline \multicolumn{2}{|l|}{} & $N=10$ \\
\hline
\end{tabular}

\section{Discussion}

By conducting two complementary studies, we aimed to answer the overarching question, whether our prototype - as an exemplary digitalization for Service Blueprinting - can effectively support the DT method. The decisive factor here is to evaluate if the prototype enables the necessary DT mindset, fits into the DT sequence, motivate users to collaboratively create service blueprints and finally, if the tool will be perceived as effective and useful.

We started a qualitative analysis in which DT experts and laymen evaluated the usability of the prototype. To not influence the participants' DT mindset all collaborative functions of the prototype 
were disabled at this point. Instead, the simple interaction with the interface was observed. As the results show, the mindset is transferred though laymen had difficulties to solve more complex tasks and kind of lost the required mindset. However, on-site Service Blueprinting also requires some experience to decide e.g. about information that needs to be included. Usually the modeler breaks down complex models into several. In a next revision of the prototype a subblueprint capability may be included. Further investigations can also focus on the identification of work cultures and the "relationships among members" [9], since these factors directly influence the mindset.

Regarding the usefulness and application in different DT phases (RQ2/H2), the test users found the prototype to be usable in both structuring information (e.g. phases Understand or Point of View) and modeling ideas and improvements (e.g. DT Prototyping). The quantitative assessment also proved the prototype's general usefulness. The extent, to what the subjects perceive the prototype as useful and userfriendly, was tested using the measurements Perceived Usefulness and Perceived Ease of Use from TAM. In addition, the measurement Value/Usefulness was taken from the IMI. The Perceived Usefulness value of the subjects is slightly increased $(M=4.894, S D=$ 1.331) and deviates significantly from the mean scale value. Additionally, the measurement Value/ Usefulness is also at a similar level $(M=4.828, S D=$ 1.356). To the same degree, this value significantly deviates from the mean value of the scale. Furthermore, the measurement Perceived Ease of Use is higher than the mean scale value $(M=5.550, S D=$ 1.095) and differs significantly from it.

As we showed for RQ3 the user tests revealed a subjective satisfaction with the prototype interaction. Still, the most complex task 3 caused resignation. In a digital environment, there are no direct moderators who can help and motivate participants like in on-site DT settings. This major finding of our study should be addressed in a in a next revision. We plan to use persuasive elements and digital moderators that can identify a lack of motivation or unintended usage and guide the user [28, 42]. However, we showed that a digital service blueprinting tool can be designed so that the tool is learnt quickly (RQ4). It also shows that by means of digital tools, users can learn the method of Service Blueprinting if the tasks are not too complex. In general, creative methods should be tested more as our results indicate transferability.

We also tested the motivation quantitatively (H1) which is expected to play an important role in the general acceptance - and particularly in DT. We used the measurement Interest/Enjoyment and Effort/ Importance from the IMI. The results for the former are overall increased $(M=4.955, S D=1.049)$ and deviate significantly from the mean value of the scale. Slightly higher $(M=5.336, S D=.941)$ lies the mean value of Effort/Importance. It also differs significantly from the mean value of the Likert-scale. Hence, the prototype motivates users intrinsically and at the same time, is perceived as user-friendly and useful. Both are decisive factors, which contribute to the acceptance of the prototype and its suitability as an alternative to analogue Service Blueprinting. Still, study I indicates a lack of motivation for more complex tasks, which needs to be studied in greater detail.

As the prototype is designed to particularly address a simultaneous and distributed collaboration we assessed the degree of collaboration quantitatively. We measured the joint effort of each participant by calculating activity points based on user contribution. The individual contributions represent the effort spent to reach the common goal, the successful creation of a service blueprint. The number of blueprint elements and the connections between these elements captured the team performance. The results show that every user was actively contributing to the team goal. The mean value of the activity points indicate that no user was free-riding or social loafing during the team performance. The group performance is constant over all ten groups. The low standard deviation indicates that every necessary element was created and that each team understood the given task to the same extent. Hence, the prototype enabled every user to actively collaborate and work on the team goal, which is an essential basis for the acceptance of our web-based prototype as a collaborative tool.

Lastly, we measured the Perceived Effectiveness to examine H3. The mean value is slightly increased ( $M$ $=4.460, S D=1.210$ ) and deviates significantly from the mean Likert-scale value. On this basis, H3 can be assumed as confirmed. Still, the main view of the blueprint editor was described as not clear enough in the free text fields which corresponds to the feedback we received in the first study. Especially with a high number of blueprint elements, the users had to scroll partially horizontally. This was felt to be ineffective, possibly affecting the later measurement results. Some users felt that the text-based communication in the chat as something too time-consuming. They suggested the integration of video or voice communication as this allows a richer experience while creating the blueprint. As stated in various media theories, advantages of communication in virtual teams can enhance collaboration $[8,37]$. The chat is suitable for an environment with more participants as voice calls can get mixed up. However, this can be used as an inspiration for further research. 
Overall, the evaluation shows that the prototype is suitable for the digital collaborative creation of service blueprints in dispersed teams [27, 41]. The prototype supports the required DT mindset, can motivate users and is perceived as useful, user-friendly and effective. However, we also identified problems with more complex tasks which have not been tested in study 2 . On the other hand, the collaborative extension may have caused a higher involvement with the tasks and the tool in comparison to study 1 . We argue that this first prototype proved to be a valid alternative to the analogue application of Service Blueprinting.

\section{Conclusion}

Based on the studies' results the developed prototype is generally suitable for the collaborative creation of service blueprints and works as a digital tool. Our paper contributes as Improvement according to the DSR Knowledge Contribution Framework [16]. We transferred a DT method to a digital application and evaluated the prototype in terms of mindset, motivation and collaborative capabilities.

The initial user tests with DT experts and laymen allowed an in-depth assessment of the suitability for DT and the interaction with the artefact using HCI evaluation approaches. In the quantitative evaluation, the subjects perceived the prototype as useful and user-friendly. The tool can motivate them intrinsically complementing the findings from the first study about the learnability. The activity data has shown that the prototype can motivate every team member to contribute to the creation of the blueprint. An effective collaboration in which every user contributed to the team performance was observed. Participants from both studies described the system as effective with further potential for improvement both in the structure of the interface (e.g. using persuasive elements) and in the communication media. Thus, the prototype can be characterized based on the evaluation as an effective approach to the digitization of the DT method of Service Blueprinting.

Even though a direct comparison between the digital and analogue execution of Service Blueprinting was not carried out, which marks an important limitation in our research, we included the assessment of DT experts in the first study who have already experienced both alternatives. The number of participants of our experiment further limits the generalization of our results.

However, with our developed prototype an important basis was created to better understand the digitalization potentials of DT methods. The findings can be used to further optimize the prototype as discussed above. As next steps, it is also necessary to examine how digital blueprints can be further processed within a DT method kit (e.g. integration into other methods of the process). Our paper and the developed prototype lay the groundwork for further investigations in the realm of IT-enabled innovation and DT [23]. As the mindset and the motivation to participate - especially in a digitized approach - are essential and have not yet been studied thoroughly, this paper contributes both to research on IT-enabled innovation and DT as well as to practice since the prototype is designed for the application in practice eventually.

\section{Acknowledgement}

This study is part of the project "Design Thinking for Industrial Services (DETHIS)" funded by the Federal Ministry of Education and Research (BMBF) of Germany; grant 02K14A140.

\section{References}

[1] Becker, J., D. Beverungen, R. Knackstedt, M. Matzner, O. Müller, and J. Pöppelbuß, "Bridging the Gap Between Manufacturing and Service Through IT-Based Boundary Objects", IEEE Trans. Eng. Manag. 60(3), 2013, pp. 468482.

[2] Beyhl, T., and H. Giese, "The Design Thinking Methodology at Work: Capturing and Understanding the Interplay of Methods and Techniques", In H. Plattner, C. Meinel and L. Leifer, eds., Design Thinking Research. Springer, 2016, 49-65.

[3] Bitner, M.J., A.L. Ostrom, and F.N. Morgan, "Service Blueprinting: A Practical Technique for Service Innovation”, Calif. Manag. Rev. 50(3), 2008, pp. 66-94.

[4] Bland, M., An introduction to medical statistics, Oxford University Press, Oxford, 2000.

[5] Davis, F.D., A Technology Acceptance Model for Empirically Testing New End-user Information Systems: Theory and Results, MIT, 1985.

[6] Davis, F.D., "Perceived Usefulness, Perceived Ease of Use, and User Acceptance of Information Technology", MIS Quart. 13(3), 1989, pp. 319-340.

[7] Deci, E.L., and R.M. Ryan, "Intrinsic Motivation Inventory (IMI)”, 2005. selfdeterminationtheory.org/intrinsic-motivation-inventory/ [8] Dennis, A.R., R.M. Fuller, and J.S. Valacich, "Media, Tasks, and Communication Processes: A Theory of Media Synchronicity", MIS Quart. 32(3), 2008, pp. 575-600.

[9] Dennis, A.R., and J.S. Valacich, "Rethinking Media Richness: Towards a Theory of Media Synchronicity", HICSS 1999 Proc., 1999, 1017.

[10] Dennis, A.R., J.S. Valacich, T. Connolly, and B.E. Wynne, "Process Structuring in Electronic Brainstorming", Inf. Sys. Research 7(2), 1996, pp. 268-277.

[11] Diehl, M., and W. Stroebe, "Productivity loss in brainstorming groups: Toward the solution of a riddle", $J$. Personality and Social Psych. 53(3), 1987, pp. 497-509. 
[12] Gackenheimer, C., Introduction to React, Apress, New York, 2015.

[13] Gallupe, R.B., A.R. Dennis, W.H. Cooper, J.S. Valacich, L.M. Bastianutti, and J.F. Nunamaker, "Electronic Brainstorming And Group Size", Academy of Manag. J. 35(2), 1992, pp. 350-369.

[14] Gera, S., G. Aneeshkumar, S. Fernandez, G. Gireeshkumar, I. Nze, and U. Eze, "Virtual teams versus face to face teams: A review of literature", IOSR J. Bus. and Manag. 11(2), 2013, pp. 1-4.

[15] Gibson, C.B., and J.L. Gibbs, "Unpacking the Concept of Virtuality: The Effects of Geographic Dispersion, Electronic Dependence, Dynamic Structure, and National Diversity on Team Innovation”, Administ. Sci. Quart. 51(3), 2006, pp. 451-495.

[16] Gregor, S., and A.R. Hevner, "Positioning and Presenting Design Science Research for Maximum Impact", MIS Quart. 37(2), 2013, pp. 337-355.

[17] Gumienny, R., S. Hampel, L. Gericke, M. Wenzel, and C. Meinel, "Transferring traditional design work to the digital world: does it work", Proc. Design Research Soc. Int. Conf., 2012.

[18] Hevner, A.R., S.T. March, J. Park, and S. Ram, "Design Science in Information Systems Research", MIS Quart. 28(1), 2004, pp. 75-105.

[19] Hewett, T.T., R. Baecker, S. Card, et al., ACM SIGCHI Curricula for HCI, ACM, New York, 1992.

[20] Jordan, P.W., An Introduction To Usability, CRC Press, 1998.

[21] Karau, S.J., and K.D. Williams, "The effects of group cohesiveness on social loafing and social compensation", Group Dynamics: Theory, Research, and Practice 1(2), 1997, pp. 156-168.

[22] Khalid, H.M., and M.G. Helander, "Customer Emotional Needs in Product Design", Concurrent Eng. 14(3), 2006, pp. 197-206.

[23] Lattemann, C., S. Stieglitz, and S. Kupke, "Deutsche Unternehmen auf dem Weg zum Web 2.0?”, HMD Praxis der Wirtschaftsinformatik 46(3), 2009, pp. 18-26.

[24] Lumpe, J., K. Purkhardt, A. Muller, D. Cravero, E. Chentnik, and T. Mott, Developing a Redux Edge, Bleeding Edge Press, 2016.

[25] Mayring, P., "Qualitative content analysis: theoretical foundation, basic procedures and software solution", 2014.

[25] Mendes, I. do N., and A.C. Dias-Neto, "A ProcessBased Approach to Test Usability of Multi-platform Mobile Applications", Design, User Experience, and Usability: Design Thinking and Methods, Springer, 2016, 456-468.

[27] Meske, C., T. Brockmann, K. Wilms, and S. Stieglitz, "Social Collaboration and Gamification", In S. Stieglitz, C. Lattemann, S. Robra-Bissantz, R. Zarnekow and T. Brockmann, eds., Gamification. Springer, 2017, 93-109.

[26] Meske, C., and T. Potthoff, "The DINU-Model - A Process Model for the Design of Nudges", ECIS 2017 Proc., 2017, 2587-2597.

[29] Nunamaker, J.F., N.C. Romano, and R.O. Briggs, Collaboration Systems: Concept, Value, and Use, M.E.Sharpe, 2014.

[30] Patrício, L., R.P. Fisk, J. Falcão e Cunha, and L. Constantine, "Multilevel Service Design: From Customer Value Constellation to Service Experience Blueprinting", $J$.
Service Research 14(2), 2011, pp. 180-200.

[29] Paulhus, D.L., and S. Vazire, "The self-report method", In R.W. Robins, R.C. Fraley and R.F. Krueger, eds., Handbook of research methods in personality psychology. Guilford Press, New York, 2007, 224-239.

[32] Plattner, H., C. Meinel, and L. Leifer, Design Thinking Research: Making Design Thinking Foundational, Springer, 2016.

[33] Pretlow, C., and K. Sobel, "Rethinking Library Service: Improving the User Experience with Service Blueprinting", Public Services Quart. 11(1), 2015, pp. 1-12.

[32] Randrup, N., D. Druckenmiller, and R.O. Briggs, "Philosophy of Collaboration", HICSS 2016 Proc., 2016.

[33] Redlich, B., D. Siemon, C. Lattemann, and S. RobraBissantz, "Shared Mental Models in Creative Virtual Teamwork", HICSS 2017 Proc., 2017.

[34] Ryan, R.M., and E.L. Deci, "Self-determination theory and the facilitation of intrinsic motivation, social development, and well-being", Amer. Psychologist 55(1), 2000, pp. 68-78.

[35] Schouten, A.P., B. van den Hooff, and F. Feldberg, "Virtual Team Work: Group Decision Making in 3D Virtual Environments", Communic. Research, 2013, pp. 180-210.

[38] Shostack, G.L., "Designing services that deliver", $H B R$ (Jan-Feb), 1984, pp. 133-139.

[39] Somech, A., and A. Drach-Zahavy, "Translating Team Creativity to Innovation Implementation The Role of Team Composition and Climate for Innovation", J. Manag. 39(3), 2013, pp. 684-708.

[40] Stickdorn, M., and J. Schneider, This is Service Design Thinking: Basics - Tools - Cases, BIS Publishers, Amsterdam, 2011.

[41] Stieglitz, S., and S. Hassannia, "Idea Generation by Employees and External Participants in Innovation Competitions", HICSS 2016 Proc., 2016, 4272-4281.

[39] Strohmann, T., D. Siemon, and S. Robra-Bissantz, "brAInstorm: Intelligent Assistance in Group Idea Generation", Int. Conf. Design Sci. Research Inform. Sys., Springer 2017, 457-461.

[43] Venkatesh, V., M.G. Morris, G.B. Davis, and F.D. Davis, "User Acceptance of Information Technology: Toward a Unified View", MIS Quart. 27(3), 2003, pp. 425478.

[41] Walny, J., S. Carpendale, N.H. Riche, G. Venolia, and P. Fawcett, "Visual thinking in action: visualizations as used on whiteboards", IEEE Trans. Vis. Comput. Graphics 17(12), 2011, pp. 2508-2517.

[45] Weerawardena, J., and F.T. Mavondo, "Capabilities, innovation and competitive advantage", Industrial Marketing Manag. 40(8), 2011, pp. 1220-1223.

[46] Wenzel, M., L. Gericke, C. Thiele, and C. Meinel, "Globalized Design Thinking: Bridging the Gap Between Analog and Digital for Browser-Based Remote Collaboration", In H. Plattner, C. Meinel and L. Leifer, eds., Design Thinking Research. Springer, 2016, 15-33.

[47] West, J., A. Salter, W. Vanhaverbeke, and H. Chesbrough, "Open innovation: The next decade", Research Policy 43(5), 2014, pp. 805-811.

[48] Zupan, B., and A.S. Nabergoj, "Developing Design Thinking Skills in Entrepreneurship Education", Leading Through Design, 2012, pp. 525. 